\title{
A Colorimetric Interdental Probe as a Standard Method to Evaluate Interdental Efficiency of Interdental Brush
}

\author{
D. Bourgeois ${ }^{1, *}$, F. Carrouel ${ }^{2,3}$, J.C. Llodra ${ }^{2,4}$, M. Bravo ${ }^{4}$ and S. Viennot ${ }^{1}$ \\ ${ }^{1}$ Laboratory "Health, Individual, Society" EA4129, University Lyon1, France; ${ }^{2}$ Faculty of Odontology, University \\ Lyon1, France; ${ }^{3}$ IGFL, UMR5242, Lyon, France; ${ }^{4}$ Department of Preventive and Community Dentistry, Faculty of \\ Odontology, University of Granada, Spain
}

\begin{abstract}
The aim of this study is to evaluate the concordance between the empirical choice of interdental brushes of different diameters compared to the gold standard, the IAP CURAPROX ${ }^{\mathcal{O}}$ calibrating colorimetric probe. It is carried out with the aim of facilitating the consensus development of best practices. All the subjects' interproximal spaces were evaluated using the reference technique (colorimetric probe), then after a time lapse of $1.2 \pm 0.2$ hours, using the empirical clinical technique (brushes) by the same examiner. Each examiner explored 3 subjects. The order the patients were examined with the colorimetric interdental probe (CIP) was random. 446 sites were selected in the study out of 468 potential sites. The correspondence of scores between interdental bushes $v$. colorimetric probe is $43.0 \%$ [95\%-CI: $38.5-47.6]$. In $33.41 \%$ of the 446 sites, the brush is inferior to the probe; in $23.54 \%$ of cases, the brush is superior to the probe. Among the discrepancies there is thus a tendency for the subjects to use brushes with smaller diameter than that recommended by the colorimetric probe. This review has found very high-quality evidence that colorimetric probes plus interdental brushing is more beneficial than interdental brushing alone for increase the concordance between the empirical choice of interdental brushes of different diameters compared to the gold standard. Uncertainties remain and further research is required to provide detailed data on user satisfaction.
\end{abstract}

Keywords: Assessment, calibration, colorimetric probe, interdental brush, grading.

\section{INTRODUCTION}

Effective oral hygiene is a crucial factor in maintaining good oral health, which is associated with overall health and health-related quality of life [1]. The control of biofilm is the common pillar of the prevention and control of periodontal disease. Better understanding of oral physiopathology has led to the introduction of the more modern concept of the disruption of biofilm instead of the elimination of dental plaque $[2,3]$. Dental hygiene is conventionally ensured by the twice-daily use of a suitable toothbrush with the aim of controlling the accumulation of supragingival plaque $[4,5]$. Various brushing techniques have been described to suit the dexterity of the patient, the anatomy of the gingival complex and the periodontal condition. Manual and electric toothbrushes of various designs are available to meet these requirements [6].

However brushing alone is not sufficient to reach and maintain a high level of oral hygiene in the medium and long term [7-10]. Independently of the length of brushing and the technique used, this relative insufficiency is mainly due to the inaccessibility of the interproximal spaces, which account for as much as a third of the surfaces accessible and/or covered by biofilm [11]. The effective cleaning of interdental spaces using toothbrushes is a challenge [12].

\footnotetext{
*Address correspondence to this author at the University Lyon 1, 11 rue Guillaume Paradin 69372 Lyon cedex 08, France; Tel: +33 4787786 84; E-mail: denis.bourgeois@univ-lyon1.fr
}

The removal of interproximal biofilm is considered to be important for the maintenance of gingival health, prevention of periodontal disease and the reduction of caries. As an adjunct to brushing, the interdental brush (IDB) currently represents the primary methods available for interproximal cleaning [13]. The majority of the studies presented a positive significant difference in the plaque index when using the IDB compared with floss [14]. Even if there was insufficient evidence to determine whether interdental brushing reduced or increased levels of plaque when compared to flossing [1], interdental brushes (IDBs) are more effective in removing plaque as compared with brushing alone or the combination use of tooth brushing and dental floss [15].

The choice of size of interdental brush suitable for the morphology of the mouth is empirical, with risk of undersizing - with impact on efficiency - and oversizing - with impact on acceptability, efficiency and gum trauma. Similarly, accessibility of the areas to be cleaned is a basic criterion for motivating users. The anatomy of interproximal spaces is very variable between healthy individuals and in the same individual - depending on the morphology of the tooth and the papilla [16]. These spaces can also change with age, periodontal health or dental treatment. Originally prescribed for large interdental spaces [1], IDBs were subsequently recommended for mixed and narrow interdental spaces on account of the wide variety of diameters available [1]. The basic premise is to use available brushes which easily fit the interdental space and whose bristles can reach the dental surfaces to disrupt the biofilm. This theoretical concept is difficult to translate into clinical practice because of the dif- 
ficulty of recommending a reference method for the choice of an IDB suitable for the interdental space.

With so many empirical choices and IDB sizes, the marketing of a colorimetric probe of progressive diameter based on the WHO community periodontal index of treatment needs (CPITN) probe model, would seem a useful aid to deciding on the optimal sizing of interdental brushes. The concordance between the results of the empirical method, taught and used in clinical practice, and those of an initial screening of clinically healthy young adults with calibrated colorimetric probes, deserves to be analysed.

The aim of this study is to evaluate the concordance between the empirical choice of interdental brushes of different diameters compared to the gold standard, the IAP CURA$\mathrm{PROX}^{\mathcal{C}}$ calibrating colorimetric probe. It is carried out with the aim of facilitating the consensus development of best practices.

\section{MATERIALS AND METHODOLOGY}

This was a non-interventional comparative cross-section study with iterative measurements. It comprised three steps: i) training of practitioners in the use of IAP CURAPROX ${ }^{(}$ colorimetric probes, ii) collecting of full-mouth interdental site diameters with the colorimetric probe, iii) matched and deferred collecting of interdental diameters provided by the gauge of CURAL ${ }^{\circledR}$ wire core of the interdental brushes.

\section{Study Population}

Recruitment and examination of the subjects were performed at the University School of Dental Medicine, Lyon, France. The study involved six clinical General Dental Practitioners (GDPs) including two private dental practitioners, a public health dentist and three dental school faculty members. The criteria for inclusion of the subjects were: age 25 to 35 years, healthy from periodontal point of view (pockets less than $2 \mathrm{~mm}$ ), declaring at least two tooth brushings per day, with no clinically significant dental anomalies or prosthetic restoration, and accepting the study terms of reference. Periodontal biotype was not considered as an inclusion criteria. The following were excluded: subjects at risk of infection or major haemorrhage, and those with immunosuppression, diabetes, haemophilia, those taking anti-platelet or anticoagulant agents and/or those with a history of periodontal illness or treatment, and subjects undergoing a course of dental treatment. Six subjects were selected following screening and interview.

\section{Internal Validity}

The examiners were trained beforehand in the use of the IAP CURAPROX ${ }^{\mathcal{C}}$ probe. A procedure manual was developed and distributed to the study examiners in advance of the training session. A PowerPoint ${ }^{\odot}$ presentation was used for training. A gold standard examiner (DB), specialist in epidemiology, and with vast experience in screening, led calibration stages. All examiners obtained a minimum kappa value of 0.84 compared to the gold standard examiner.

\section{Empirical and Comparative Technique}

There is no agreed scientific method concerning the choice of interdental brushes [17]. The so-called reference technique is the empirical subjective method in everyday use. This consists in testing the various IDBs in increasing order of diameter. The IDB which fits the interdental space with friction thought 'sufficient but not excessive' by the operator is chosen as the best suited for the interdental space in question.

The IDBs used are from the CPS range of CURAPROX $^{\odot}$. This pack comprises 5 cylindrical IDBs with the following characteristics:

- A colour code relating to the size of the brush

- An access diameter defined by the gauge of the CURAL ${ }^{\circledR}$ wire core used for stiffening the IDB

- An effective cleaning diameter defined by the length of the synthetic bristles covering the working part of the device.

The characteristics of the IDBs are resumed in the following Table 1:

Table 1. Characteristics of the IDBs.

\begin{tabular}{|c|c|c|c|c|c|}
\hline Colour code & Blue (B) & Red (R) & Pink (P) & $\begin{array}{c}\text { Yellow } \\
(\mathbf{Y})\end{array}$ & $\begin{array}{c}\text { Green } \\
(\mathbf{G})\end{array}$ \\
\hline \hline Access diameter (mm) & 0.6 & 0.7 & 0.8 & 0.9 & 1.1 \\
\hline $\begin{array}{c}\text { Effective cleaning diameter } \\
(\mathrm{mm})\end{array}$ & 2.2 & 2.5 & 3.2 & 4.0 & 5.0 \\
\hline
\end{tabular}

\section{Technical Reference}

Calibration of the interdental diameter is made by means of a dedicated probe. The IAP CURAPROX ${ }^{\mathcal{C}}$ calibrating probe is a graduated conical instrument with a rounded tip (Fig. 1). The working part comprises coloured bands from the point to the base corresponding to IDBs by increasing diameter. The largest section of each coloured band corresponds to the cleaning efficiency diameter of the relevant brush. The non-working part has a click-fastening joint for the attachment of a handle for easier use and access to the interproximal spaces at the back of the mouth.

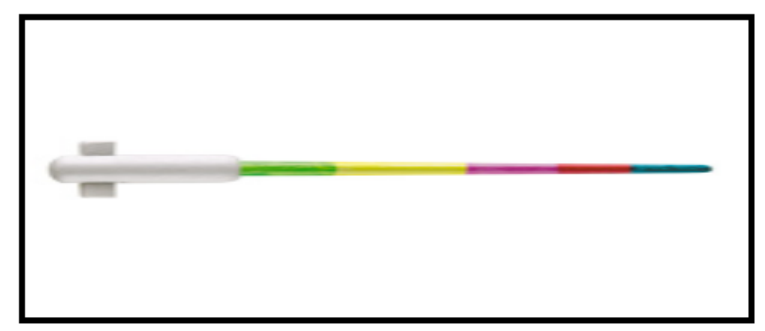

Fig. (1). IAP CURAPROX ${ }^{\mathscr{O}}$ probe.

Interdental diameters were coded as " 1 - Blue -, 2 - Red, 3 - Pink, 4- Yellow and 5- Green." The procedure consists in introducing the IAP CURAPROX ${ }^{\odot}$ probe into the vestibular interdental space, inserting it fully, then noting the colour emerging from the interdental space on the vestibular side. This corresponds to the colour code of the IDB most suitable for the space in question. The pressure used to place the 
probe tip at the base of the interdental sites was approximately $50 \mathrm{~N} / \mathrm{cm}^{2}$ (0.20 gram force). CURAPROX@ prime instruments used in this study are not exclusively focused on healthy patients but it can be argued that the target for this is the choice of range of IDB's products.

\section{Clinical Examination}

All the subjects' interproximal spaces, except those between the $2^{\text {nd }}$ and $3^{\text {rd }}$ molars, were evaluated using the reference technique (colorimetric probe), then after a time lapse of at least one hour, using the empirical clinical technique (brushes) by the same examiner. Each examiner explored 3 subjects. The order the patients were examined with the colorimetric interdental probe (CIP) was random. The statistical unit was the interdental space. 446 sites were retained in the study out of 468 potential sites ( 26 sites/subject x 3 subjects x 6 examiners). This difference is linked to the absence of 5 first premolars in subjects ( 5 sites $\times 3$ examiners $=15$ ), diastema is present in 2 sites and 1 site excluded due to a incorrect clinical examination by 1 examiner (no space instead code 1). The large number of sites tested by each operator guarantees the variability of site sizes and a large number of repetitions. The use of the interdental brushes was carried out at a sufficiently long time interval to avoid the operator remembering the results of the previous test but sufficiently short to guarantee the compatibility of the operational conditions. The time between the initial CIP screening administration and the IDB baseline retest was $1.2 \pm 0.2$ hours. Meticulous visual inspection with a good operation light was recommended. The results of the tests were entered by a third party on a separate record by test and by subject. They were not consultable by the operator during the procedure. The analysis of the data was done blind.

\section{Analysis}

The interdental site was taken as the unit of analysis, with the main purpose of evaluating the concordance between dental colorimetric probe (the reference standard direct visual method) and IDB data. The analyses were performed globally, i.e., all sites together, and stratifying by location of sites: Molar (16-17, 26-27, 36-37 and 46-47), Premolar (15$16,14-15,13-14,25-26,24-25$ and $23-24$; 35-36, 34-35, $33-$ $34,45-46,44-45,43-44)$, and Incisor (22-23, 21-22, 21-11, $11-12$ and $12-13 ; 32-33,31-32,31-41,41-42,42-43)$. We considered the five categories of interdental score as an ordinal scale, e.g., $1(0.6 \mathrm{~mm}), 2(0.7 \mathrm{~mm}), 3(0.8 \mathrm{~mm}), 4(0.9$ $\mathrm{mm}), 5(1.1 \mathrm{~mm})$.

Different approaches were followed to valuate the association between probe and IDB data: Spearman's rank correlation, comparison of their distribution with Wilcoxon paired rank test, and quadratic weighted kappa as a measure of concordance for ordinal scales [18]. Kappa values can be interpreted as follows: $j$ value $0.00-0.20$ poor agreement; $j$ value 0.21-0.40 fair agreement; $j$ value $0.41-0.60$ moderate agreement; $j$ value 0.61-0.80 good agreement, and $j$ value 0.81 1.00 excellent agreement.

Furthermore, we estimated the change (distance or difference) between probes and brushes in their ordinal scale for a given site as the number of jumps, i.e., 0 when probe and brush are the same, +1 if the ordinal value of the probe is one level higher than brush, and so on. Then, we estimated percents and $95 \%$-CIs of those changes between probes and brushes.

SPSS 15.0 (SPSS Inc., Chicago, IL) was used for the statistical analysis. $\mathrm{P}$ values smaller than 0.05 were considered statistically significant.

\section{RESULTS}

\section{Overall ID Detection Rate and Type Distribution}

The overall detection rate and type distribution of probes and brushes in ordinal colorimetric scale is shown in Table 2. Using the probe, a score $\leq 2$ is obtained in $43.3 \%$ (193/446) of the sample vs. $48.9 \%$ for brush. Among the 446 sites, $37.5 \%$ (167/446) were coded with $\geq 4$ CIP score vs. 37.9 for brush.

Table 3 shows the distribution of the IDB scores at screening versus probe values. In relation to the agreement between probe and brushes, we observe a wide variation depending on the score. We observe that the $\%$ of sites correctly classified in relation to the gold standard of the probe is very different depending on the score and varies between $15.1 \%$ (for score 4 ) and $75.5 \%$ (for score 5 ). It is also interesting to note how the disagreements are distributed depending on the score. Independently of the score, the tendency to underestimate (choose a brush smaller than indicated by the probe) is more frequent than the reverse situation (larger brush than indicated by the probe).

In Table 4 we observe that a global correlation exists $\mathrm{r}=0.71$ between the probe and the brushes. The quadratic weighted kappa values are over 0.65 , both at the global analysis level and for the analysis by dental location (molars, premolars and incisors), which indicates a concordance which should be considered substantial according to the Landis \& Koch scale.

The correspondence of scores between interdental brushes $v$ s. colorimetric probe is $43.0 \%$ [95\%-CI: 38.5-47.6]. In $33.41 \%$ of the 446 sites, the brush is inferior to the probe; in $23.54 \%$ of cases, the brush is superior to the probe. Among the discrepancies there is thus a tendancy for the subjects to use brushes with smaller diameter than that recommended by the colorimetric probe (Table 5).

\section{DISCUSSION}

Industry has responded well to the oral health needs of the public through the development of new products and technologies [20]. New preventive products have been developed and recommended by professional to protect sites at risk [21]. Dental floss has been used for many years in conjunction with brushing for removing dental plaque in between teeth. Also, interdental brushes have been developed which many people find easier to use than floss, providing there is sufficient space between the teeth [1].

The relevant outcome scale for CIP used for the choice and indication of IDBs is a novel observer rating instrument recently developed for daily clinical practice to allow dentists and other oral health teams to determine the accuracy of 
Table 2. Description of Probes and Brushes in ordinal colorimetric scales. ( $n=446$ exploration sites).

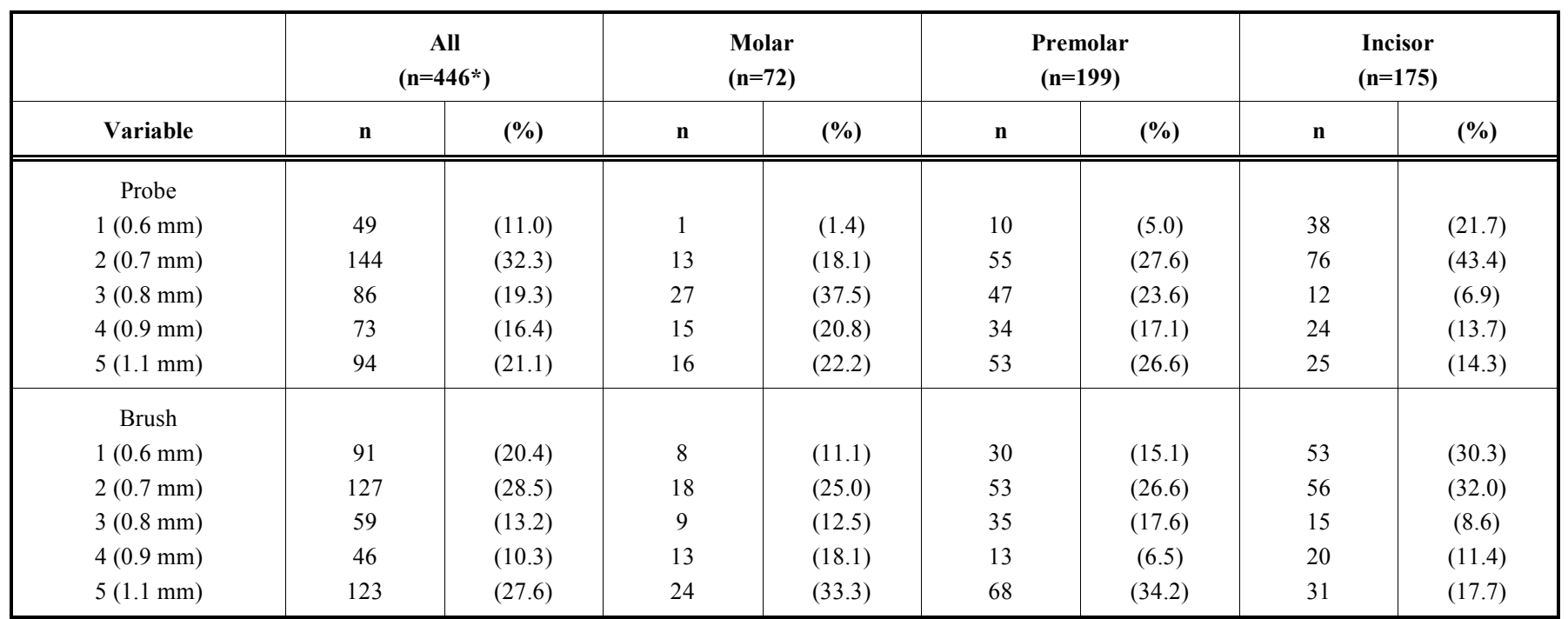

(*) Only patients with no missing values for both assessments were used for the test-retest reliability analysis (9 sites)

Table 3. Associations between Probes and corresponding Brushes in ordinal colorimetric scale. ( $\mathrm{n}=446$ exploration sites).

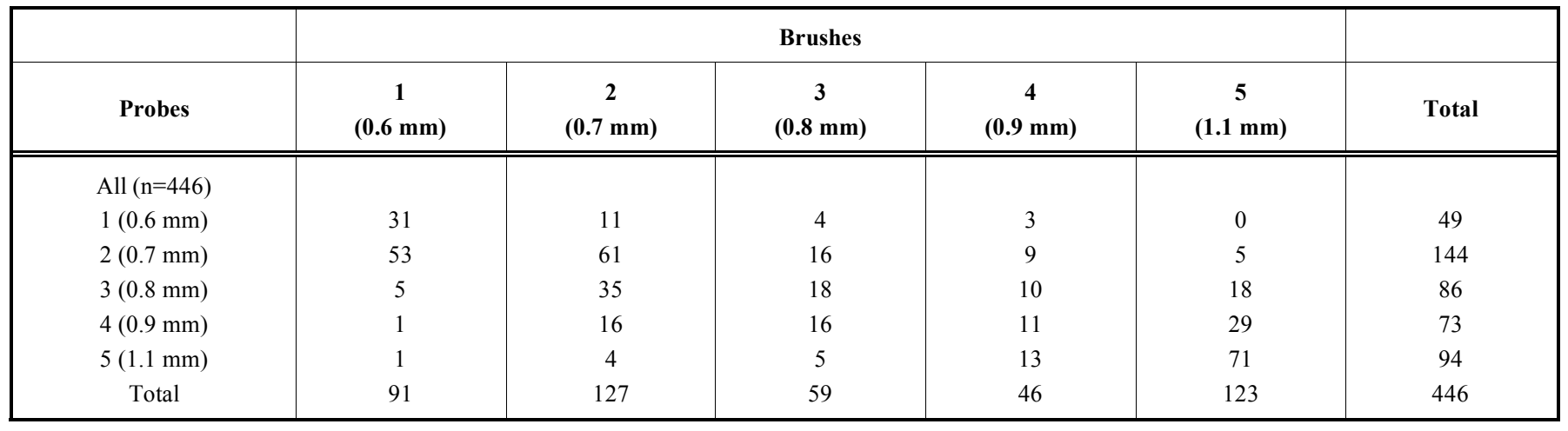

Number of exploration sites in each cell.

Table 4. Associations (analytic) between Probes and corresponding Brushes. ( $n=446$ exploration sites).

\begin{tabular}{|c|c|c|c|}
\hline Probe versus Brush & Correlation $^{\mathrm{a}}$ & Comparison $^{\mathrm{b}}$ & Concordance $^{\mathbf{c}}$ \\
\hline \hline All $(\mathrm{n}=446)$ & $\mathrm{r}_{\mathrm{s}}=0.71, \mathrm{p}<0.001$ & $\mathrm{p}=0.158$ & Q-W-Kappa $=0.70(95 \%-\mathrm{CI}=0.65-0.75)$ \\
\hline Molar $(\mathrm{n}=72)$ & $\mathrm{r}_{\mathrm{s}}=0.70, \mathrm{p}<0.001$ & $\mathrm{p}=0.569$ & $\mathrm{Q}-\mathrm{W}-\mathrm{Kappa}=0.67(95 \%-\mathrm{CI}=0.52-0.78)$ \\
\hline Premolar $(\mathrm{n}=199)$ & $\mathrm{r}_{\mathrm{s}}=0.69, \mathrm{p}<0.001$ & $\mathrm{p}=0.089$ & $\mathrm{Q}-\mathrm{W}-\mathrm{Kappa}=0.67(95 \%-\mathrm{CI}=0.59-0.74)$ \\
\hline Incisor $(\mathrm{n}=175)$ & $\mathrm{r}_{\mathrm{s}}=0.66, \mathrm{p}<0.001$ & $\mathrm{p}=0.954$ & Q-W-Kappa $=0.70(95 \%-\mathrm{CI}=0.61-0.76)$ \\
\hline
\end{tabular}

a: Spearman's rank correlation $\left(r_{s}\right)$

b: Wilcoxon paired rank test.

c: Quadratic weighted kappa, which can be evaluated against the Landis \& Koch scale i.e. poor $(<0.00)$, slight $(0.00-0.20)$, fair $(0.21-0.40)$, moderate $(0.41-0.60)$, good $(0.61-0.80)$, and excellent (0.81-1.00) [19].

relevant efficiency diameter of IDBs of patients and to document prevention progression and care effects over time. As such, there is a need for new techniques/devices to be developed that will make interdental cleaning easier and improve patient motivation [22]. The following requirements were determined for an ideal practical scale: easy and quick administration; high reliability and validity; behaviour, communication, quality of life, relevance for all ID severity stages; suitability for long-term monitoring oral health conditions [23, 24].

The main research question addressed by this assessment is "What is the clinical effectiveness of interdental brush based on healthy periodontal people" in a context where the evidence base for clinical effectiveness of IDB is very mixed. 
Table 5. Change between Probes and Brushes (number of jumps). ( $n=446$ exploration sites).

\begin{tabular}{|c|c|c|c|c|c|c|c|c|c|c|c|c|}
\hline \multirow[b]{2}{*}{ Number of jumps } & \multicolumn{3}{|c|}{$\begin{array}{c}\text { All } \\
(n=446)\end{array}$} & \multicolumn{3}{|c|}{$\begin{array}{l}\text { Molar } \\
(n=72)\end{array}$} & \multicolumn{3}{|c|}{$\begin{array}{c}\text { Premolar } \\
(n=199)\end{array}$} & \multicolumn{3}{|c|}{$\begin{array}{l}\text { Incisor } \\
(n=175)\end{array}$} \\
\hline & $\mathbf{n}$ & $(\%)$ & 95\%-CI & $\mathbf{n}$ & (\%) & $95 \%$-CI & $\mathbf{n}$ & $(\%)$ & 95\%-CI & $\mathbf{n}$ & $(\%)$ & 95\%-CI \\
\hline \multicolumn{13}{|l|}{ Number of jumps } \\
\hline-3 & 8 & $(1.8)$ & $0.8-3.5$ & 1 & (1.4) & $0.0-7.5$ & 3 & $(1.5)$ & $0.3-4.3$ & 4 & $(2.3)$ & $0.6-5.7$ \\
\hline-2 & 31 & $(7.0)$ & $4.8-9.7$ & 5 & (6.9) & $2.3-15.5$ & 14 & $(7.0)$ & $3.9-11.5$ & 12 & $(6.9)$ & $3.6-11.7$ \\
\hline+1 & 117 & $(26.2)$ & $22.2-30.3$ & 21 & $(29.2)$ & $19.0-41.1$ & 56 & $(28.1)$ & 21.9-34.4 & 40 & $(22.9)$ & $16.6-29.1$ \\
\hline+2 & 26 & $(5.8)$ & $3.8-8.4$ & 4 & $(5.6)$ & $1.5-13.6$ & 13 & $(6.5)$ & $3.5-10.9$ & 9 & $(5.1)$ & $2.4-9.5$ \\
\hline+3 & 5 & $(1.1)$ & $0.4-2.6$ & 0 & $(0.0)$ & $0.0-5.0$ & 4 & $(2.0)$ & $0.6-5.1$ & 1 & $(0.6)$ & $0.0-3.1$ \\
\hline+4 & 1 & $(0.2)$ & $0.0-1.2$ & 0 & $(0.0)$ & $0.0-5.0$ & 0 & $(0.0)$ & $0-1.8$ & 1 & $(0.6)$ & $0.0-3.1$ \\
\hline \multicolumn{13}{|l|}{ Valid correspondence } \\
\hline
\end{tabular}

\section{The Limitations of this Study}

Authors have deliberately chosen to use and report simpler, descriptive statistical measures for this study because they are widely used and easily computed. Weighted kappa is most appropriately used in the assessment of reliability for ordered classifications but this measure should be interpreted with caution not only in light of the weighting issue but because it behaves more like a measure of association than an index of agreement [18]. Different formulation exist, but with slightly different descriptors [19, 25]. The choice of benchmarks, however, is inevitably arbitrary, and the effects of prevalence and bias on kappa must be considered when judging its magnitude [26]. When weighted kappa is used, the choice of weighting scheme will affect its magnitude. The larger the number of scale categories, the greater the potential for disagreement, with the result that unweighted kappa will be lower with many categories than with few [26]. If quadratic weighting is used, however, kappa increases with the number of categories, and this is most marked in the range from 2 to 5 categories [27].

In our study, kappa values exceeded 0.65 , both for all the sites and for different dental locations. The prevalence of ID scores (IDS) observed at sessions did not vary very dramatically. The content validity analyses demonstrated a good correlation between the CIP and each of the validated scales used as standard measures for the assessment of IDS.

The examination presents many limitations in its use: it requires subjective evaluations to be made by the practitioner; access diameter can go undetected because teeth are typically examined by the naked eye. The readings may also be influenced by several factors such as calculus, plaque and prophylactic pastes, and no consistent cleaning procedures $[7,28]$. Therefore, detection by eyesight is better at an advanced stage than early and presents many limitations related to the experience of the examiner. This is particularly true since our model is applicable to clinically healthy subjects. Consequently, diagnosis of the ID space by visual inspection is partial and auxiliary methods are needed as adjunct to conventional examination for identifying and quantifying such ID space [29]. The CIP adds a qualitative plus value insofar as there is no agreed scientifically established method for the choice of IDBs. The observation capacity of the CIP should guide the clinician toward a more preventive and minimal care strategy with monitoring space progression over time and not tempt him/her to overrecommend an IDB.

In contrast, with CIP system, the space and its real topography can be seen in a magnified enlarged view. Due to that "external visibility" of the space, the interpretation of the effective cleaning diameter is easier especially in the posterior sectors of the mouth. However, our study has not highlighted qualitative differences between differently localised sectors. When comparing the measurements for both methods, our results demonstrated a substantial agreement among the two techniques. These results indicated similarity in diagnosis among the observers. In the current study, CIP was set as "gold standard" due to validated relationship between its codes and the physiological diameter of the IDBs. No studies have shown good reproducibility and accuracy of technical instruments for cleaning efficiency diameter detection in permanent teeth especially in the case where subjects are without periodontal lesions [28]. This would make it appropriate to use component criteria, i.e. CIP approach, for grading to judgment scores which can be made more effective, more acceptable and less traumatic than the empirical and subjective method used in current practice with interdental brushes.

\section{Clinical Considerations}

Thus the key question is the criterion of choice of the IDB, and secondly, the quality of the user's technique. The baseline screening made with visual examination with probe is well correlated with the diagnosis made by IDB. The evaluation is the same irrespective of the location of the sites (Molar, Premolar and Incisor) with no statistical difference.

The level of reliability, as measured by the quadratic weighted kappa, is not dependent on the classification of diameter's scale used as the outcome measure. This is particularly interesting in the context where the majority of examiners are practising dentists, are not employed full time as 
study examiners and have divergent educational qualifications and clinical experience.

The effectiveness of IDBs on the reduction of gingival inflammation is considerable, but the relevance of their use by healthy patients is debated [1]. Two criteria need to be taken into consideration. The possibility of inserting an IDB in spaces considered clinically healthy. Our study indicates that the probe of $0.6 \mathrm{~mm}$ diameter with a 0.20 gram force $(50$ $\mathrm{N} / \mathrm{cm}^{2}$ ) is able to penetrate all sites without difficulty, so it could be considered as a preventive factor in the disruption of the interdental pellicle. The main problem with all interdental cleaning is, however, patient ability and motivation. Interdental cleaning does not readily become an established part of daily oral hygiene. As such, CIPs can be considered as a newly developed technique/device that will make interdental cleaning easier and improve patient motivation. An indirect advantage of introducing a calibrated brush which does not require multiple passes is to avoid damage to the interdental papilla and prevent any abrasive trauma to the dental surfaces. From a clinical point of view, the prophylactic goal to achieve high degrees of cleaning while producing minimal damage, due to the side effects and misuse of interdental brushes, is important. It is necessary to emphasize individual instruction and selection of means for oral hygiene with a view to attaining a high level of cleanliness and thereby doing little harm to both soft (recessions, loss of papillae) and hard tissues (abrasion).

The efficient cleaning diameter $(\mathrm{mm})$ needs to be taken into consideration. The dental pellicle plays a fundamental role in bacterial colonisation and the formation of biofilm $\left(10^{8}\right.$ and $\left.10^{9} \mathrm{bacteria} / \mathrm{mg}[11]\right)$, whatever the state of the individual as regards caries and/or periodontal disease. So, it would be logical to combine the modern molecular biology technologies with microbiology to be able to quantify the concept of efficient cleaning and/or disruption of dental biofilm. Associating the IDBs from the CPS range of CURAPROX $^{\odot}$ which have an access diameter defined by the thickness of the wire core serving as reinforcement, and this access diameter is correlated to a cleaning efficiency diameter (from $2.2 \mathrm{~mm}$ to $5.0 \mathrm{~mm}$ ), with the initial use of a dedicated IAP type calibrating probe minimises the risk of bias in the interpretation of the efficiency diameter.

\section{Implication for Practice}

This review has found very high-quality evidence that colorimetric probes plus interdental brushing is more beneficial than interdental brushing alone to increase the concordance between the empirical choice of interdental brushes of different diameters compared to the gold standard. Clinically, the discordances noted in the study indicate that in $23.54 \%$ of cases, the brushes chosen have a diameter larger than that indicated by the probe, whereas in $33.41 \%$ the opposite is the case (brush with diameter smaller than the probe value). Using the pressure standardisation possibilities of the CIP, it is clear that the empirical method of choosing brushes leads in almost a quarter of cases to excessive pressure through choosing a diameter wider than that recommended by the probe. Some extreme were noted (though infrequent) situations: in 17 cases a brush from codes 4-5 was used, when the CIP recommended brushes 1 or 2 .
Interdental (ID) screening in young adults without disease may detect different states, potentially leading to a reduction in disease symptoms and in the medium term to increased quality-adjusted life expectancy. The prophylactic goal to achieve high degrees of intercleaning while producing minimal damage is important and should have priority when evaluating and selecting IDB.

Screening ID space with CIP for IDB should be clinically beneficial and comes at a cost-effectiveness ratio superior to other accepted interventions in oral health care. No study has assessed the cost-effectiveness of ID screening interventions in asymptomatic patients. The colorimetric interdental probes screening was effective and probably cost-effective in comparison to the usual practice for increasing interdental brushing in daily oral health activity. It may have the potential to be increasingly used for prevention and control by healthy periodontal people. The screening of periodontal healthy subjects is important as is shown by the fact that the CIP was able to specify an interdental brush.

\section{CONCLUSION}

Daily interdental cleaning is important for the maintenance of gingival health, prevention of periodontal disease and the reduction of caries. The CIP is an efficiency instrument of choice in order to a suitable IDB.

\section{CONFLICT OF INTEREST}

The authors confirm that this article content has no conflict of interest.

\section{ACKNOWLEDGEMENTS}

Declared none.

\section{AUTHORS' CONTRIBUTIONS}

$\mathrm{DB}, \mathrm{FC}$ was the coordination investigator of the clinical trial and provided critical remarks on the manuscript. DB, JC developed the first draft of this article based on extensive discussions with all other authors. FC, SV contributed to planning and MBP execution of the statistical analyses. All authors read and approved the final manuscript.

\section{REFERENCES}

[1] Poklepovic T, Worthington HV, Johnson TM, et al. Interdental brushing for the prevention and control of periodontal diseases and dental caries in adults. Cochrane Database Syst Rev 2013; 12 : CD009857.

[2] Palmer RJ Jr. Composition and development of oral bacterial communities. Periodontol 2000 2014; 64: 20-39.

[3] Zijnge V, van Leeuwen MBM, Degener JE, et al. Oral biofilm architecture on natural teeth. PloS One 2010; 5: 9321.

[4] Bourgeois DM, Phantumvanit P, Llodra JC, Horn V, Carlile M, Eiselé JL. Rationale for the prevention of oral diseases in primary health care: an international collaborative study in oral health education. Int Dent J 2014; 64:1-11.

[5] Rosema NA, Timmerman MF, Versteeg PA, et al. Comparison of the use of different modes of mechanical oral hygiene in prevention of plaque and gingivitis. J Periodontol 2008; 79: 1386-94.

[6] Deacon SA, Glenny AM, Deery C, van Palenstein HWH, Van der Velden U, Van der Weijden GA. Different powered toothbrushes for plaque control and gingival health. Cochrane Database Syst Rev 2010; 12: CD004971. 
[7] Slot D, Wiggelinkhuizen L, Rosema N, Van der Weijden G. The efficacy of manual toothbrushes following a brushing exercise: a systematic review. Int J Dent Hyg 2012; 10: 187-97.

[8] Biesbrock AR, Walters PA, Bartizek RD. The relative effectiveness of six powered toothbrushes for dental plaque removal. J Clin Dent 2002; 13: 198-202.

[9] Rosema N, Slot DE, van Palenstein HW, Wiggelinkhuizen L, Van der Weijden G. The efficacy of powered toothbrushes following a brushing exercise: a systematic review. Int J Dent Hyg 2014; doi: 10.1111/idh.12115.

[10] Slot DE, Wiggelinkhuizen L, Rosema NA, Van der Weijden GA. The efficacy of manual toothbrushes following a brushing exercise: a systematic review. Int J Dent Hyg 2012; 10: 187-97.

[11] Rosan B, Lamont RJ. Dental plaque formation. Microbes Infect Inst Pasteur 2000; 2: 1599-607.

[12] Vogel M, Sener B, Roos M, Attin T, Schmidlin PR. Interdental cleaning and gingival injury potential of interdental toothbrushes. Swiss Dent J 2014; 124: 1290-301.

[13] Sälzer S, Slot DE, Van der Weijden FA, Dörfer CE. Efficacy of interdental mechanical plaque control in managing gingivitis meta-review. J Clin Periodontol 2015; 42(16): 92-105.

[14] Slot DE, Dörfer CE, Van der Weijden GA. The efficacy of interdental brushes on plaque and parameters of periodontal inflammation: a systematic review. Int J Dent Hyg 2008; 6: 253-64.

[15] Gluch JI. As an adjunct to tooth brushing, interdental brushes (IDBs) are more effective in removing plaque as compared with brushing alone or the combination use of tooth brushing and dental floss. J Evid Based Dent Pract 2012; 12: 81-3.

[16] Wolff D1, Joerss D, Dörfer CE. In vitro-cleaning efficacy of interdental brushes with different stiffness and different diameter. Oral Health Prev Dent 2006; 4: 279-85.
[17] Claydon NC. Current concepts in toothbrushing and interdental cleaning. Periodontol 2000 2008; 48: 10-22.

[18] Fleiss JL. Statistical methods for rates and proportions. $2^{\text {nd }}$ ed. New York, NY: John Wiley \& Sons Inc 1981.

[19] Landis JR, Koch GG. The measurement of observer agreement for categorical data. Biometrics 1977; 33: 159-74.

[20] Alexander D, Clarkson J, Buchanan R, et al. Exploring opportunities for collaboration between the corporate sector and the dental education community. Eur J Dent Educ 2008; 12 (Suppl 1): 64-73.

[21] Addy M. Oral hygiene products: potential for harm to oral and systemic health? Periodontol 2000 2008; 48: 54-65.

[22] Warren PR, Chater BV. An overview of established interdental cleaning methods. J Clin Dent 1996; 7(3): 65-9.

[23] Matthews DC. Prevention and treatment of periodontal diseases in primary care. Evid Based Dent 2014; 15: 68-9.

[24] Podschun GD. National plan to improve health literacy in dentistry. J Calif Dent Assoc 2012; 40: 317-20.

[25] Altman DG. Practical Statistics for Medical Research. $1^{\text {st }}$ ed. London, England: Chapman \& Hall/CRC 1991.

[26] Brennan P, Silman A. Statistical methods for assessing observer variability in clinical measures. BMJ 1992; 304: 1491-4.

[27] Brenner H, Kliebsch U. Dependence of weighted kappa coefficients on the number of categories. Epidemiology 1996; 7: 199202.

[28] Chongcharoen N, Lulic M, Lang NP. Effectiveness of different interdental brushes on cleaning the interproximal surfaces of teeth and implants: a randomized controlled, double-blind cross-over study. Clin Oral Implants Res 2012; 23: 635-40.

[29] Novaes TF, Matos R, Celiberti P, Braga MM, Mendes FM. The influence of interdental spacing on the detection of proximal caries lesions in primary teeth. Braz Oral Res 2012; 26: 293-9.

Received: February 13, 2015

Revised: August 31, 2015

Accepted: September 29, 2015

(C) Bourgeois et al.; Licensee Bentham Open.

This is an open access article licensed under the terms of the (https://creativecommons.org/licenses/by/4.0/legalcode), which permits unrestricted, noncommercial use, distribution and reproduction in any medium, provided the work is properly cited. 